\title{
Status of the ANTARES project
}

\author{
J. Brunner ${ }^{a}$ on behalf of the ANTARES collaboration

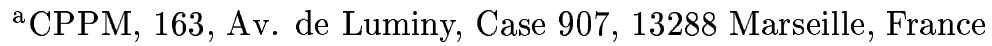

The ANTARES collaboration is building a neutrino telescope in the Mediterranean sea close to the French coast. The project aims to detect atmospheric and extraterrestrial neutrinos with energies above $10 \mathrm{GeV}$ by means of the Cherenkov light that is generated in water by charged particles which are produced in neutrino interactions. The project and its physics potential will be described and the construction status will be illustrated.

\section{Introduction}

The ANTARES collaboration has been set up in 1996. Today it involves groups from France, United Kingdom, Spain, Italy, The Netherlands, Russia and Germany. From 1996 to 1999 an extensive $R \& D$ program had been successfully performed to prove the feasibility of the detector concept. The environment parameters at various deep sea sites have been studied and a deployment site of the experiment has been chosen[1]. It is $10 \mathrm{~km}$ south of the Hyeres archipelago at $42^{\circ}$ $50^{\prime} \mathrm{N}, 6^{\circ} 10^{\prime} \mathrm{E}$. It combines the advantage of an important depth of $2475 \mathrm{~m}$ with the vicinity to the coast and infrastructure (harbors of Toulon and La Seyne).

\section{Detector design}

After the successful R\&D phase the construction of the ANTARES detector has been decided in 2000 and is progressing. The detector consists of 12 lines and a junction box which distributes the power and clock synchronization signals to the lines and collects the data. The junction box is connected to the shore by a $42 \mathrm{~km}$ electrooptical cable. The lines have an equipped vertical length of $350 \mathrm{~m}$ starting $100 \mathrm{~m}$ above sea floor. Their horizontal distance is about $65 \mathrm{~m}$ and they are arranged to form a regular octagon on the sea floor. Each line is connected to the junction box with the help of a submarine using wet-mateable connectors. It is composed of 25 storeys with a vertical distance of $14.5 \mathrm{~m}$. The lines are kept

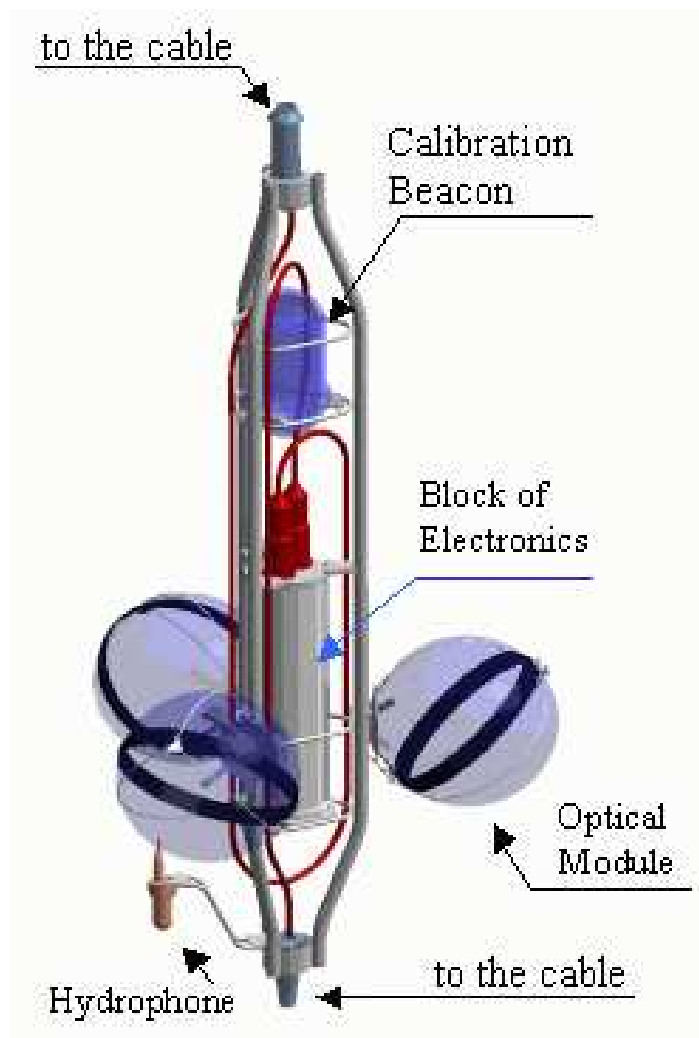

Figure 1. Key element of the detector: The storey 
straight by the floating force of a buoy at the top and an anchor at the bottom. They float in the sea current and the positions of the active detector elements are permanently monitored by an acoustic calibration system.

Each storey (see Fig. 1) contains three $45^{\circ}$ downward looking 10" photomultipliers inside pressure resistant glass spheres - the optical modules (OM) [2]. The electronic cards are inside a titanium cylinder at the center. Some of the storeys contain supplementary calibration equipment like acoustic or optical beacons.

The signals of each photomultiplier are readout by two ASICs. For simple pulses charge and arrival time are digitized and stored for transfer to the shore station. For more complex pulses the pulse shape can be digitized with $1 \mathrm{GHz}$ sampling frequency. The time stamps are synchronized by a clock signal which is sent in regular intervals from the shore to all electronic cards. The overall time calibration is better than $0.5 \mathrm{nsec}$. Therefore the time resolution of the signal pulses will be limited by the transition time spread of the photomultipliers $(\sigma \sim 1.3 \mathrm{nsec})$. All data are sent to the shore station. With a noise light rate of $70 \mathrm{kHz}$ on the one photon level this produces a data flow of $1 \mathrm{Gbit} / \mathrm{sec}$ to the shore. In the shore station a PC farm performs a data filtering to reduce the data rate by at least a factor 100 .

\section{Construction status}

From November 1999 to June 2000 a "demonstrator line" had been operated to prove the feasibility of the foreseen project. Its most important result was the verification that the acoustic position system is able to locate each OM with a precision of $5 \mathrm{~cm}$ with respect to a grid of fix points on the sea floor.

In October 2001 the final electro-optical cable was deployed over a length of $42 \mathrm{~km}$ from the foreseen ANTARES site to La Seyne where the power station and the control room are located.

In December 2002, the junction box was connected to the remote cable end and was deployed. Since its deployment it is permanently monitored and has flawlessly functioned.

During the same period a first prototype line

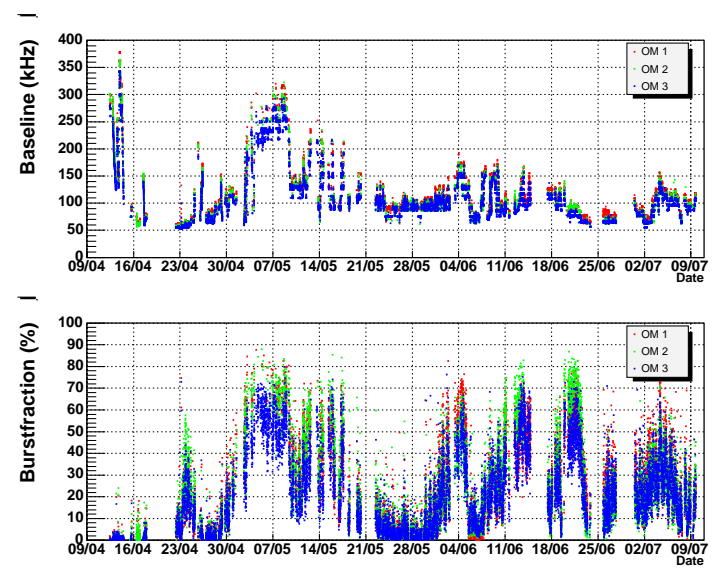

Figure 2. Monitoring of single photoelectron counting rates during the three months operation of the prototype line in 2003. Signals from all three OMs of one storey are shown. Above: Baseline rates averaged over 5 minutes. Below: Fraction of time for which the measured rate is significantly higher than the baseline rate (burst fraction)

had been assembled. It represents $1 / 5$ of a full line, 5 storeys with 15 OMs. Extensive tests of this line have been performed. The most important result was the verification of the timing accuracy which can be reached by the system. The time resolution of the pulses has been confirmed to be $1.3 \mathrm{nsec}$ at the single photoelectron level.

In December 2002 the prototype line was deployed followed by another short line which contained supplementary devices to monitor environment parameters (sea currents, sound velocity, salinity, water transparency) or to serve as calibration elements for the prototype line (laser and LED beacons).

In March 2003 both lines were successfully connected to the junction box by the manned submarine 'Nautile'. The prototype line took continuously data for three months until its recovery in July 2003. One of its results is the measurement of the counting rates of the photomultipliers which is illustrated in figure 2 . 
Meanwhile the mass production for the full scale detector has started. The ANTARES detector should be fully operational in 2007 .

\section{Physics performance}

Most studies so far concentrated on charged current interactions of $\nu_{\mu}$ :

$\nu_{\mu}\left(\bar{\nu}_{\mu}\right)+N \rightarrow \mu^{-}\left(\mu^{+}\right)+X$

The direction of the muon is reconstructed using the fact that it emits Cherenkov light under a well defined angle and does not suffer from multiple scattering at high energies. In ANTARES several reconstruction algorithms for muons have been developed. They use the direct Cherenkov hits but take also into account secondary effects like diffusion, dispersion and electromagnetic showers which accompany high energetic muons. This leads to an angular resolution for the muons of better than $0.2^{\circ}$ above $1 \mathrm{TeV}$ for the above mentioned $1.3 \mathrm{nsec}$ single pulse resolution. To obtain the neutrino angular resolution one has to consider also the interaction kinematics and gets $0.7^{\circ}$ at $1 \mathrm{TeV}$ which decreases to the detectordominated $0.2^{\circ}$ at $100 \mathrm{TeV}$.

The estimation of the neutrino energy is based on the measurement of the light output of the muon track in the vicinity of the detector. In the $\mathrm{TeV}$ range the light output increases with energy due to radiative processes. However the measurement is compromised by the facts that these radiative processes are stochastic, the neutrino interaction point is invisible in most cases and only a short fraction of the muon track is seen in the detector. Nevertheless procedures have been found which estimate the neutrino energy within a factor 3 for energies below $100 \mathrm{TeV}$ and within a factor 2 for higher energies.

The effective area is another important parameter which characterizes the performance of the detector.

Fig. 3 gives the effective areas for a neutrino flux before interaction and before penetration of the Earth for various nadir angle bins. This has the advantage that such an effective area can be folded directly with neutrino flux predictions from astronomical sources to obtain signal events

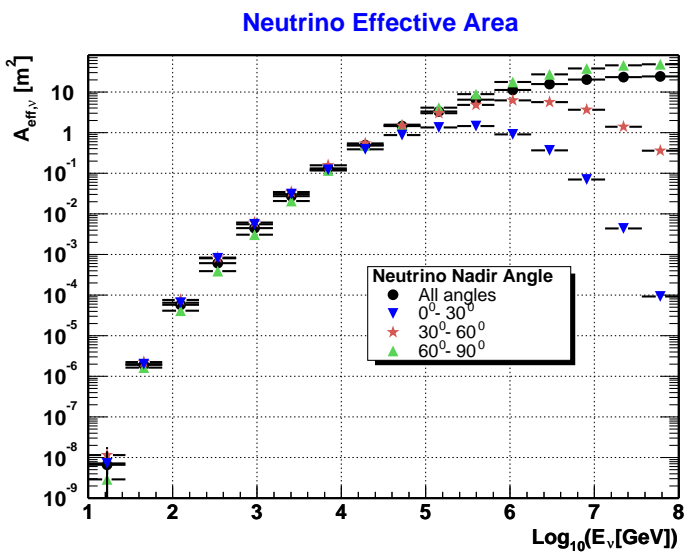

Figure 3. Energy dependence of the effective area for neutrinos before entering the Earth

and it can be easily compared to effective areas of gamma ray telescopes. There are three differences with respect to the usually shown effective areas for muons: the overall scale changes from $\mathrm{km}^{2}$ to $\mathrm{m}^{2}$ due to the smallness of the neutrino cross section; the energy dependence becomes much stronger due to the almost linear raise of the neutrino cross section; the opacity of the Earth limits the effective area to values below $20 \mathrm{~m}^{2}$.

Using the above performance parameters one can estimate that ANTARES will detect about 3000 upward going muon tracks from atmospheric neutrinos per year. They are the major background for the search for diffuse astronomical neutrino fluxes. The only distinction is their supposedly harder spectrum. With an energy cut of $50 \mathrm{TeV}$ a flux $E^{2} \Phi(E)$ of $10^{-7} \mathrm{GeV} \mathrm{cm}{ }^{-2} \mathrm{~s}^{-1} \mathrm{sr}^{-1}$ can be excluded after one year of data taking assuming $\Phi(E) \sim E^{-2}$. For the search for point like sources an energy cut is a priori not needed. Cluster algorithms and likelihood methods have been developed to identify unknown point sources on an isotropic background of tracks from atmospheric neutrinos. After one year an $\mathrm{E}^{-2}$ flux of $4-22 \cdot 10^{-16} \mathrm{~cm}^{-2} \mathrm{~s}^{-1}$ (depending on declination) can be tested for declination angles of the source smaller than $40^{\circ}$. This remains the biggest dis- 
covery potential of ANTARES complementary to South pole experiments which can be nicely demonstrated with microquasars. These objects are promising Galactic neutrino sources. Flux estimates have been given in [3]. The microquasar SS433 is a permanent source which is visible for AMANDA and ANTARES. For AMANDA in one year of observation (365 days active) 2 signal events are expected for an estimated background of 4.4 [4] whereas for ANTARES 4.3 signal events are expected with 0.3 background events from atmospheric neutrinos assuming a conservative angular cut of 1 degree around the source position. Another permanent microquasar (invisible for AMANDA) is GX339 which would yield a signal of 6.5 events in Antares according to [3] with the same background. Other potential sources are supernovae remnants and the Galactic center. Recently the gamma ray telescope HESS reported the observation of a very hard flux $\left(E^{-2.2}\right)$ from SNR RX J1713.7-3946 [5], as well as from Sag $A^{*}[6]$. Both sources are well visible for ANTARES.

The search for dark matter is another important physics subject apart from astronomy. Weakly interacting massive particles (WIMPS) are candidates for dark matter. The lightest supersymmetric particle $\chi$ is stable in R-parity conserving models and therefore a possible candidate for such a WIMP. They could be gravitationally captured in the center of astronomical bodies until an equilibrium between capture rate and annihilation rate is reached. The annihilation produces conventional particles which subsequently produce also neutrinos in their decay chain. Fig. 4 shows the sensitivity of ANTARES after three years of data taking for a signal from the Sun.

\section{Conclusion}

The construction of the ANTARES detector has started. Data from the complete detector can be expected in 2007. The expected sensitivity for point-like neutrino sources and WIMPS searches will allow significant contributions in these research fields and might lead to exciting results in the near future.

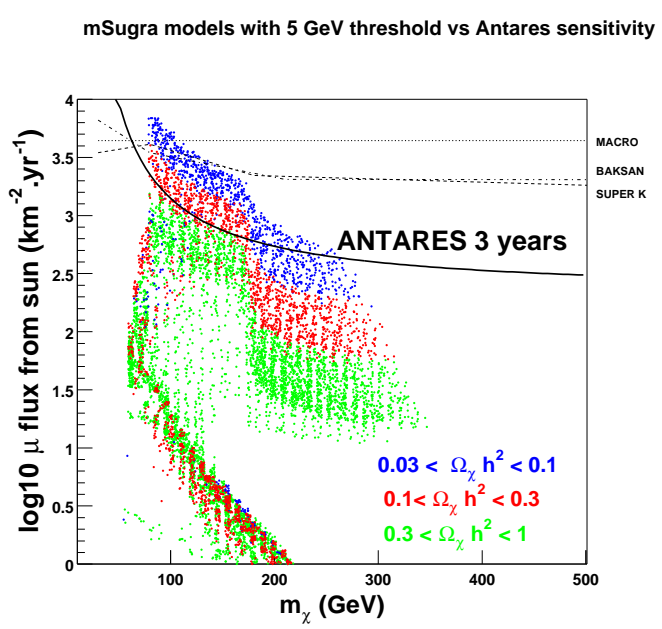

Figure 4. Limits for muon flux from the direction of the Sun due to annihilation of WIMPS, existing limits [7-9] and expectations from mSUGRA models are also indicated

\section{REFERENCES}

1. P. Amram et al. (ANTARES collaboration), Astropart. Phys. 13 (2000) 127.

2. P. Amram et al. (ANTARES collaboration), Nucl. Instr. Meth. A484 (2002) 369.

3. C. Distefano, D. Guetta, E. Waxmann and A. Levinson, Astrophys. J. 575 (2002) 378.

4. J. Ahrens et al. (AMANDA collaboration), Phys. Rev. Lett. 92 (2004) 071102.

5. F. A. Aharonian et al. (HESS collaboration), Nature 432 (2004) 75.

6. F. Aharonian et al. (HESS collaboration), arXiv:astro-ph/0408145.

7. M. Ambrosio et al. (MACRO collaboration), Phys. Rev. D60 (1999) 082002.

8. O. V. Suvorova et al. (BAKSAN collaboration), arXiv:hep-ph/9911415.

9. S. Desai et al. (SUPERKAMIOKANDE collaboration), Phys. Rev. D70 (2004) 083523. 